\title{
A recombined fusion protein PTD-Grb2-SH2 inhibits the proliferation of breast cancer cells in vitro
}

\author{
JIKAI YIN $^{1^{*}}$, ZHONGLIANG CAI $^{2 *}$, LI ZHANG $^{3}$, JIAN ZHANG $^{4}$, \\ XIANLI HE ${ }^{1}$, XILIN DU ${ }^{1}$, QING WANG ${ }^{1}$ and JIANGUO LU ${ }^{1}$ \\ ${ }^{1}$ Department of General Surgery, Tangdu Hospital of The Fourth Military Medical University, Ba'qiao, Xi'an, Shaanxi 710038; \\ ${ }^{2}$ Research Institute of General Surgery, Nanjing General Hospital of Nanjing Military Command, Xuan'wu, Nanjing, \\ Jiangsu 210002; ${ }^{3}$ Department of Ultrasound Diagnosis, Tangdu Hospital of The Fourth Military Medical University, \\ Ba'qiao, Xi'an, Shaanxi 710038; ${ }^{4}$ State Key Laboratory of Cancer Biology, Department of Biochemistry and \\ Molecular Biology, The Fourth Military Medical University, Ba'qiao, Xi'an, Shaanxi 710032, P.R. China
}

Received November 4, 2012; Accepted December 17, 2012

DOI: $10.3892 /$ ijo.2013.1768

\begin{abstract}
The growth factor receptor bound protein 2 (Grb2) is one of the affirmative targets for cancer therapy, especially for breast cancer. In this study, we hypothesized the Src-homology 2 (SH2) domain in Grb2 may serve as a competitive proteinbinding agent to interfere with the proliferation of breast cancer cells in vitro. We designed, constructed, expressed and purified a novel fusion protein containing the protein transduction domain (PTD) and Grb2-SH2 domain (we named it after PTD-Grb2-SH2). An immunofluorescence assay was used to investigate the location of PTD-Grb2-SH2 in cells. MTT assay and EdU experiments were applied to detect the proliferation of breast cancer cells. The ultra-structure was observed using transmission electron microscopy. Flow cytometry was used to determine the cytotoxicity of PTD-Grb2-SH2 on cell proliferation. We successfully obtained the PTD-Grb2-SH2 fusion protein in soluble form using a prokaryotic expression system. The new fusion protein successfully passed through both the cellular and nuclear membranes of breast cancer cells. The MTT assay showed that PTD-Grb2-SH2 exhibited significant toxicity to breast cancer cells in a dose- and time-dependent manner in vitro. EdU identified the decreased proliferation rates in treated MDA-MB-231 and SK-BR-3 cells. Observation by transmission electron microscopy and flow cytometry further confirmed the cytotoxicity as apoptosis. Our results show that the HIV1-TAT domain is a useful tool for transporting a low
\end{abstract}

Correspondence to: Dr Jianguo Lu, Department of General Surgery, Tangdu Hospital of The Fourth Military Medical University, 1 Xin'si Road, Ba'qiao, Xi'an, Shaanxi 710038, P.R. China

E-mail: lujguo@hotmail.com

${ }^{*}$ Contributed equally

Key words: breast cancer, protein transduction domain, growth factor receptor bound protein 2, Src-homology 2 domain molecular weight protein across the cell membrane in vitro. The PTD-Grb2-SH2 may be a novel agent for breast cancer therapy.

\section{Introduction}

Breast cancer is the second leading cause of cancer related death among women worldwide. It was estimated that there will be 230,480 new cases of invasive breast cancer and 39,520 new deaths among US women in 2011 (1). Among the molecular targets for treatment purpose, epidermal growth factor receptor (EGFR) and human epidermal growth factor receptor 2 (HER2) were most widely investigated as potential targets for anticancer drug development since the first report of the overexpressions in human breast cancers indicates a poor prognosis $(2,3)$. EGFR overexpression has been found in $15 \%$ of unselected, but half of triple-receptor-negative (TRN) breast cancers (4), and HER2 is amplified in 20 to $30 \%$ of breast cancers. All these changes lead to enhanced malignant phenotypes and highly aggressive disease $(5,6)$. HER2-overexpressing breast cancers also exhibited the capability of resistance to many first-line chemotherapy and tamoxifen (7-9). Moreover, many fundamental studies revealed that EGFR and HER2 have a close relationship with the aberrant activation of Ras proteins, which is implicated in facilitating virtually all aspects of the malignant phenotype, including cellular proliferation, transformation, invasion and metastasis (10).

Growth factor receptor bound protein 2 (Grb2) is one of the most important proteins participating in EGFR and HER 2 signal transduction. It consists of one Src-homology 2 (SH2) domain and surrounded by two Src-homology 3 (SH3) domains. The SH2 domain interacts with phosphotyrosines (pTyr) on target proteins, while the $\mathrm{SH} 3$ domains interact with proline-rich sequences (11). Once the membrance receptor is activated, Grb2 serves as an adaptor protein in many signal transductions including the mitogen-activated protein kinase (MAPK) cascade for promoting cell division and/or differentiation (12). Silencing the Grb2 expression reduced cell growth in vitro indicating the GRB2 protein could be a good target for cancer therapy (13). The SH2 domain is one of the most 
prevalent protein-binding modules for protein-protein interaction which mediate the formation of multiprotein complexes during signaling. $\mathrm{SH} 2$ domains specifically function in protein tyrosine kinase (PTK) pathways due to the dependence of their binding on tyrosine phosphorylation (14). In cells, the specific association of $\mathrm{SH} 2$ domains and tyrosine phosphopeptides is to mediate the reversible relocalization of proteins, which is important for efficient propagation of PTK signals (15). The specificity of $\mathrm{SH} 2$ domain has allowed these domains to function as probes to detect tyrosine phosphorylation in signaling proteins $(16,17)$. We hypothesized that Grb2-SH2 domain may serve as a negative inhibitor if it binds to an activated receptor in a living cell, but lacks SH3 domains to bind those downstream modules.

In order to investigate the possibility of our hypothesis, we utilized PTD as a tool to bring SH2 domains into living cells. The most popular PTD is HIV-1 TAT48-60 (GRKKRRQRRRPPQ), which has 12 amino acid arginine-rich peptides with the ability to rapidly translocate outside proteins into cells both in vivo and in vitro. Fusion of this PTD with proteins and peptides was proved to facilitate effective transduction of the fused cargos into cultured cells and animal tissues while preserving their biological activity $(18,19)$. In this study, we constructed, expressed, and purified a fusion protein containing an $\mathrm{SH} 2$ domain derived from Grb2 and a PTD domain, and investigated its potential functions in breast cancer cells.

\section{Materials and methods}

All chemicals were of analytical grade and purchased from commercial suppliers.

Bacterial strains, plasmids and cell lines. E. coli $[\mathrm{DH} 5 \alpha$ and BL21 (DE3)] (Invitrogen, Carlsbad, CA, USA) were cultured in Luria-Bertani broth medium (LB) and stored at $-70^{\circ} \mathrm{C}$. pET-16b was a commercial product (Novagen, Billerica, MA, USA) and stored at $-20^{\circ} \mathrm{C}$. The reconstructed plasmid pET-16b-ptd was a gift from Professor Hua Han, Department of Medical Genetics and Developmental Biology, Fourth Military Medical University (20). The breast cancer cell lines HER2-negative MDA-MB-231 and HER2-positive SK-BR-3 were preserved in the Department of Molecular Biology of the Fourth Military Medical University and was routinely cultured in Dulbecco's modified Eagle's medium (DMEM) or RPMI-1640 medium supplemented with $10 \%$ fetal bovine serum (Gibco by Invitrogen, Carlsbad, CA, USA), $100 \mathrm{U} / \mathrm{ml}$ penicillin and $100 \mu \mathrm{g} / \mathrm{ml}$ streptomycin. Cell cultures maintained at $37^{\circ} \mathrm{C}$ in a humidified $5 \% \mathrm{CO}_{2}$ atmosphere.

Expression vector construction. The Grb2-SH2 coding DNA sequence was the native sequence of Grb2-SH2 as reported in Genbank (CCDS11721.1) without any modification. Grb2-SH2 cDNA was generated by PCR using the cDNAs of human lymphocytes constructed by the Department of Medical Genetics and Developmental Biology of Fourth Military Medical University using the following primers: $\mathrm{SH} 2$ sense primer, 5'-GGA TCC CTA CCG CAG GAA TAT C-3' and SH2 anti-sense primer, 5'-CTC GAG AAA CCA CAT CCG TGG-3'. PCR products were purified using QIAquick Gel Extraction Kit (Qiagen, Hilden, Germany). Resulting PCR products were digested with BamHI and XhoI into the PGEM-T-Easy reporting plasmid and subsequently sub-cloned into digested pET-16b-ptd containing a PTD coding sequence (5'-TAT GTA TGG TAG GAA GAA ACG TCG ACA GCG TCG TCG-3') derived from HIV-1 TAT ${ }^{48-60}$ (GRKKRRQRRRPPQ) and a ten-Histidine-tag sequence for easy purification to construct the expression vector pET-16b-ptd/grb2-sh2. Meanwhile, we also designed a mutant contrast for Grb2-SH2 sequence with a loss of 60 bases in sequence using a forward primer (5'-GAA GTT CAA TTC TTT GCG GTA GGG ATC-3') and a reverse primer (5'-CCG GGG ATC CCT ACC GCA AAG AAT TG-3') and inserted into digested PGEM-T-grb2-sh2 and pET-16b-ptd/grb2-sh2. The subsequent DNA sequencing and BLAST program (http://www. ncbi.nlm.nih.gov/BLAST/) confirmed all insertions to be correct.

Protein expression and purification. The reconstructed plasmids were transformed into E. coli BL21 (DE3) and a single colony was picked and grown overnight in $5 \mathrm{ml} \mathrm{LB}$ supplemented with $100 \mu \mathrm{g} / \mathrm{ml}$ ampicillin at $37^{\circ} \mathrm{C}$ then diluted $1: 100$. Protein expression induced by $1 \mathrm{mM}$ isopropyl $\beta$-D-1-thiogalactopyranoside (IPTG) (Sigma-Aldrich, St. Louis, MO, USA). After induction for $4 \mathrm{~h}$ at $33^{\circ} \mathrm{C}$, approximately $10 \mathrm{~g}$ of wet weight cells from 1-liter culture were harvested by centrifugation at $6,000 \mathrm{x} \mathrm{g}$ for $30 \mathrm{~min}$ at $4^{\circ} \mathrm{C}$ followed by re-suspension in $60 \mathrm{ml}$ of Tris- $\mathrm{HCl}$ (50 mM, pH 8.0) containing $1 \mathrm{mM}$ phenylmethylsulfonyl fluoride (PMSF) for enzyme stability. The $E$. coli cells were then pulse-sonicated for $10 \times 1 \mathrm{~m}$ (10 sec working and $50 \mathrm{sec}$ resting on ice, $300 \mathrm{~W}$ ). The lysate was centrifuged at $15,000 \mathrm{x} \mathrm{g}$ for $30 \mathrm{~min}$ at $4^{\circ} \mathrm{C}$. The recombinant His-tagged PTD-Grb2-SH2 proteins were purified from the cell lysate using $1 \mathrm{ml}$ of HisTrap ${ }^{\mathrm{TM}} \mathrm{Ni}^{++}$charged columns (Amersham Pharmacia Biotech, Uppsala, Sweden). The lysate was dialyzed against binding buffer $(20 \mathrm{mM}$ Tris- $\mathrm{HCl}, \mathrm{pH} 7.4,500 \mathrm{mM}$ $\mathrm{NaCl}, 5 \mathrm{mM}$ imidazole) overnight at $4^{\circ} \mathrm{C}$. Subsequently, the dialyzed lysate was injected into the Ni-NTA resin column and binding for $10 \mathrm{~min}$. The unbound proteins were washed away by using 10 volumes of binding buffer. The His-tagged proteins were eluted with elution buffer $(20 \mathrm{mM}$ Tris- $\mathrm{HCl}, \mathrm{pH} 7.4$, $500 \mathrm{mM} \mathrm{NaCl}, 20 \mathrm{mM}$ imidazole). The eluate was analyzed by sodium dodecyl sulfate-polyacrylamide gel electrophoresis (SDS-PAGE) and western blot analysis. The fractions containing the purified fusion protein were pooled and dialyzed against 100 volumes of $20 \mathrm{mM}$ Tris- $\mathrm{HCl}\left(\mathrm{pH} \mathrm{7.4)}\right.$ overnight at $4^{\circ} \mathrm{C}$. The protein concentration was determined using a DC protein assay kit (Bio-Rad, Hercules, CA, USA) with a bovine serum albumin (BSA) standard. The purified protein was stored at $4^{\circ} \mathrm{C}$ for use within 1 week or at $-70^{\circ} \mathrm{C}$ for long-term storage.

SDS-PAGE and western blot analysis. SDS-PAGE was followed the procedure described by Laemmli (21). For western blot analysis, the proteins were separated by SDS-PAGE on a $15 \%$ gel and transferred onto a polyvinylidene difluoride (PVDF) membrane (Bio-Rad) in transfer buffer [25 mM Tris, $192 \mathrm{mM}$ glycine, $15 \%$ (vol/vol) methanol] at $4^{\circ} \mathrm{C}$ at $100 \mathrm{~V}$ for $30 \mathrm{~min}$. After blocking with $5 \%$ milk in phosphate-buffered saline (PBS, $50 \mathrm{mM}$ phosphate and $0.9 \% \mathrm{NaCl} ; \mathrm{pH} 7.2$ ) at room temperature for $1 \mathrm{~h}$ or overnight at $4^{\circ} \mathrm{C}$, the membrane was incubated for $1 \mathrm{~h}$ with horseradish peroxidase (HRP)-labeled antibody against the His-tag (Qiagen; 1:2,000 with 2.5\% milk in PBS). The membrane was then washed five times with PBS-T 
A

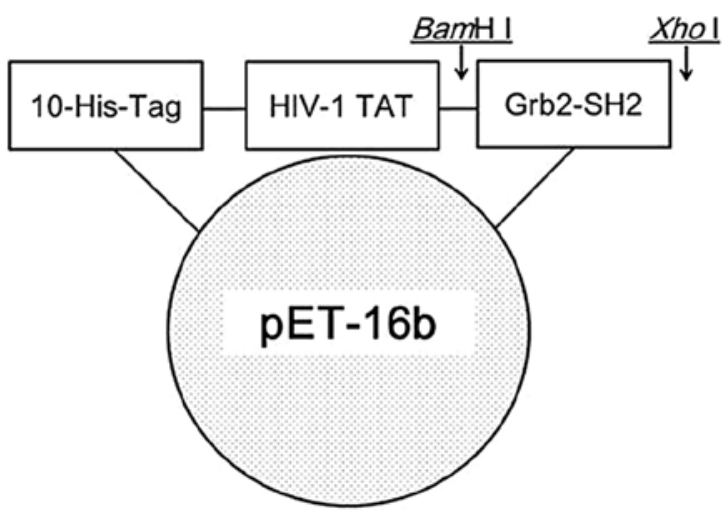

B

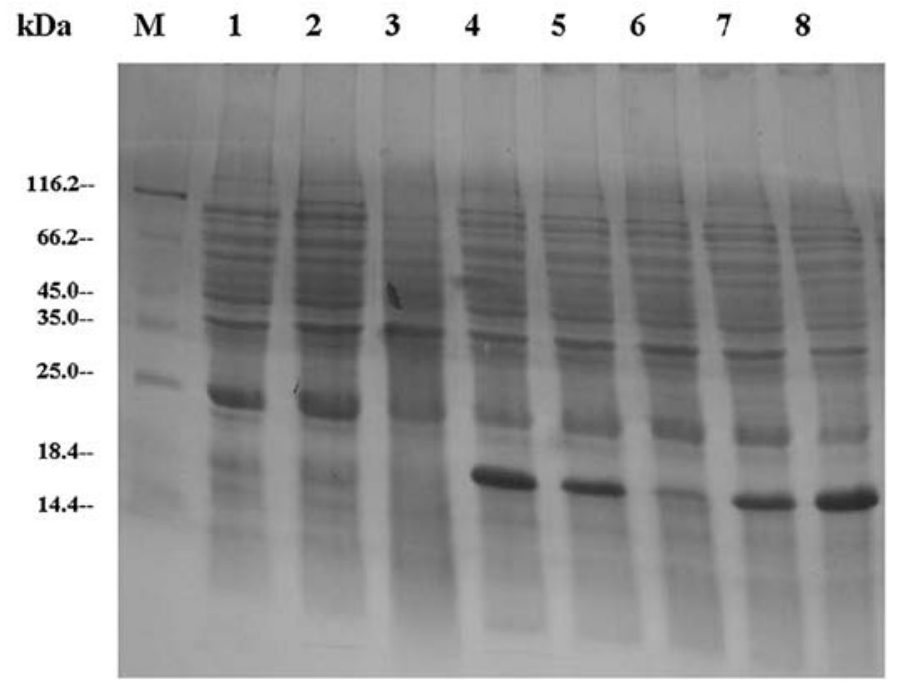

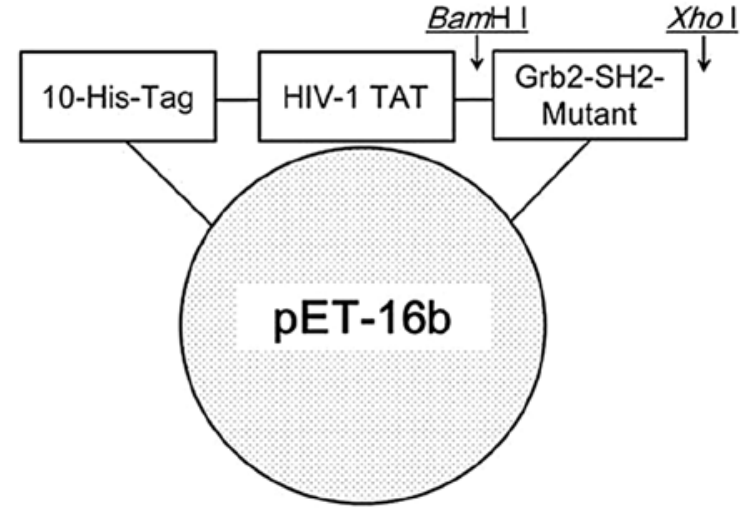

C

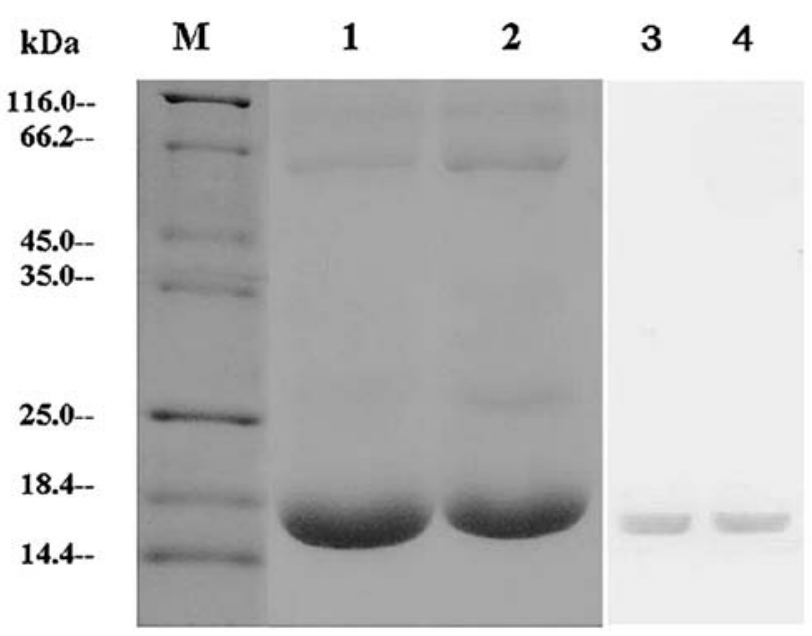

Figure 1. Construction, expression and purification of PTD-Grb2-SH2 and PTD-Grb2-SH2-Mutant. (A) The Grb2-SH2 coding frame is presented with 10 histidines and the HIV-1 TAT domain. (B) The expression of the recombinants. Lane M, protein molecular weight marker; lane 1, E. coli BL21(DE3) lysates after IPTG; lane 2, pET-16b plasmid after IPTG; lane 3, pET-16b-ptd/grb2-sh2 before IPTG; lane 4, pET-16b-ptd/grb2-sh2 recombinant after IPTG; lane 5, the crude lysate supernatant of induced pET-16b-ptd/grb2-sh2 after IPTG; lane 6, pET-16b-ptd/grb2-sh2-Mutant before IPTG; lane 7, pET-16b-ptd/grb2-sh2-Mutant after IPTG; lane 8, the crude lysate supernatant of induced pET-16b-ptd/grb2-sh2-Mutant after IPTG. (C) The purification and identification of the recombinants. Lane M, protein molecular weight marker; lane 1, the purified PTD-Grb2-SH2-Mutant fusion protein; lane 2, the purified PTD-Grb2-SH2 fusion protein; lane 3, the purified PTD-Grb2-SH2-Mutant fusion protein was identified by western blot analysis; lane 4, the purified PTD-Grb2-SH2 fusion protein was identified by western blot analysis.

(PBS plus $0.05 \%$ Tween-20) and two times with PBS. The target proteins were visualized with the enhanced chemiluminescence detection system (ECL, GE Healthcare, Piscataway, NJ, USA).

Immunofluorescence assay. Cells on cover slips were incubated with the target proteins for $4 \mathrm{~h}$. The samples were fixed in $4 \%$ paraformaldehyde, permeabilized in PBS with $0.1 \%$ Triton $\mathrm{X}-100$, blocked in $2 \%$ normal rabbit serum in $\mathrm{PBS}$, and then incubated overnight at $4^{\circ} \mathrm{C}$ with mouse anti-His polyclonal antibody (Santa Cruz Biotechnology, Santa Cruz, CA, USA) at a 1:400 dilution. Cells were extensively washed with PBS and incubated with the fluorescein-labeled rabbit anti-mouse secondary antibody (Dako, Glostrup, Denmark) at a 1:100 dilution at room temperature for $2 \mathrm{~h}$ followed by further washing. The results were assessed under a reflected light fluorescence microscope (BH2-RFC, Olympus, Tokyo, Japan).

Cell viability assay. The MTT [3-(4, 5-dimethylthiazol-2-yl)-2, 5-diphenyltetrazolium bromide] assay was used to investigate the cytotoxicity of the fusion protein. Breast cancer cells were plated in 96-well plates $\left(5 \times 10^{4}\right.$ cells per well) in septuplicate. The fusion proteins (including target protein and mutant contrast) were added into the culture medium (RPMI-1640 medium supplemented with $10 \%$ fetal bovine serum) in different concentrations $(0.05 \mathrm{mg} / \mathrm{ml}, 0.1 \mathrm{mg} / \mathrm{ml}$ or $0.2 \mathrm{mg} / \mathrm{ml})$. At the indicated time, $20 \mu \mathrm{l}$ aliquots of $5 \mathrm{mg} / \mathrm{ml}$ MTT (Sigma) in PBS were added to each well and incubated for $4 \mathrm{~h}$ followed by the addition of $150 \mu \mathrm{l}$ of $\mathrm{Me}_{2} \mathrm{SO}$. The $\mathrm{A}_{490}$ values were assayed in a Sunrise microplate reader (Tecan, Groedig, Austria). Proliferation in vitro was also determined by 5-ethynyl-2-deoxyuridine (EdU, Ribobio, Guangzhou, China). EdU incorporation was determined using a Cell-Light ${ }^{\mathrm{TM}}$ EdU Cell Proliferation Kit (Ribobio), according to the supplier's instructions. The electronic microscope was used to observe the ultrastructure of treated breast cancer cells.

Cell apoptosis analysis. Annexin V-FITC/PI staining was performed using the Elite ESP flow cytometry (FACSCalibur, Becton-Dickinson Immunocytometry Systems, San Jose, CA, USA) at $488 \mathrm{~nm}$ according to the manufacturer's guidelines. 

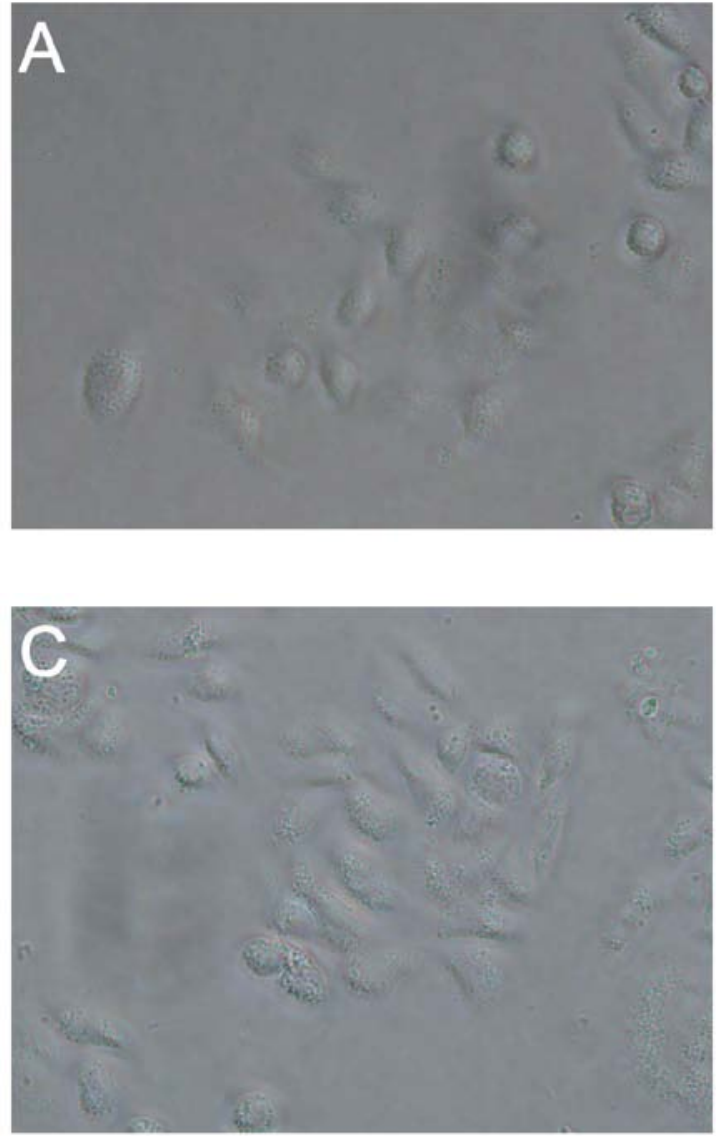
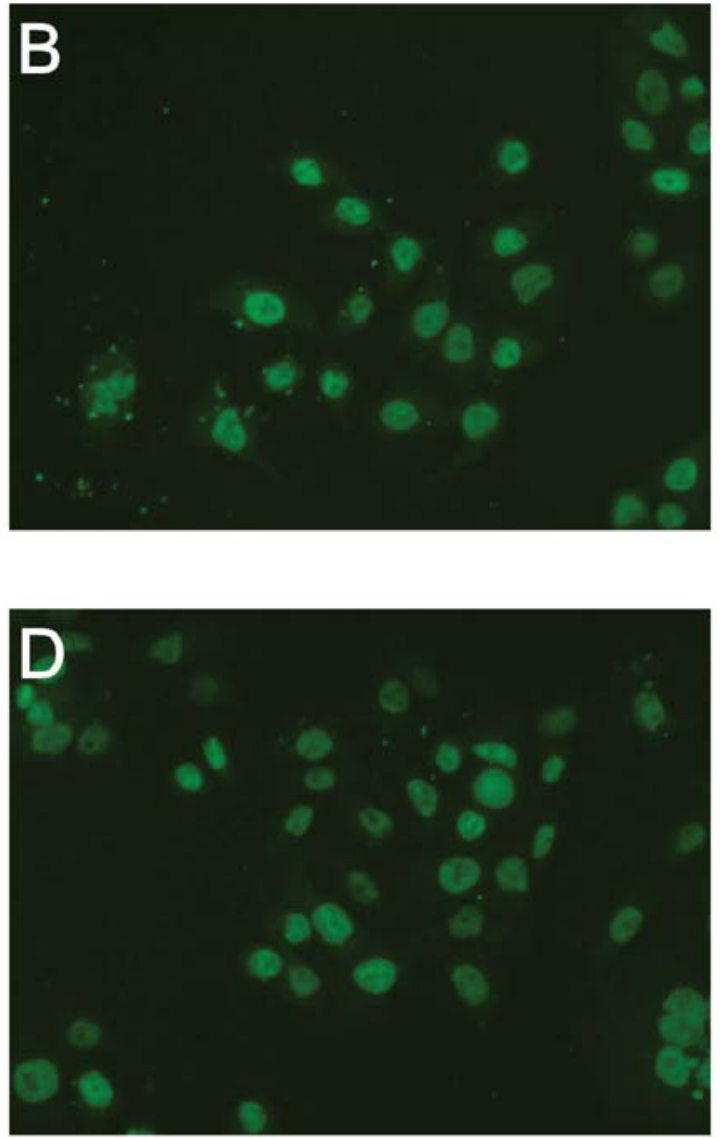

Figure 2. Transduction of the recombinants into breast cancer cell line MDA-MB-231. (A and B) Green fluorescence shows PTD-Grb2-SH2 transduced into living MDA-MB-231 cells and mainly located in the nucleus; (C and D) PTD-Grb2-SH2-Mutant successfully transduced into living cells as well.

Briefly, cells were incubated with PI and Annexin V-fluorescein isothiocynate in the darkness at room temperature. Flow cytometric analysis was immediately performed for apoptosis analysis and the data were analyzed using the Cell Quest Pro software (BD Biosciences, San Jose, CA, USA). Transmission electron microscope observation was carried out with the assistance of the Laboratory Center of Electron Microscope from Fourth Military Medical University.

Statistical analysis. Statistical analysis was performed using the SPSS 13.0 software package for Windows.

\section{Results}

Sequence synthesis, cloning, expression, purification and identification. Using the recombinant DNA technology, the PCR amplification fragments consistent with that expected for Grb2-SH2 (310 bp) and Grb2-SH2-Mutant (250 bp) were detected on an agarose gel and the DNA sequences were confirmed by automatic sequencing. The successful construction of the expressed recombinant pET-16b-ptd/grb2-sh2 and pET-16b-ptd/grb2-sh2-Mutant was confirmed by restriction mapping (Fig. 1A). In the pET system, target genes are positioned downstream of the bacteriophage T7 late promoter. Typically, products contain a prophage ( $\lambda$ DE3) encoding the highly processive T7 RNA polymerase under control of the IPTG-inducible lacUV5 promoter that ensures tight control of recombinant gene basal expression. SDS-PAGE showed the expression of soluble proteins with a molecular mass value consistent with that expected for PTD-Grb2-SH2 (17.6 kDa) and PTD-Grb2-SH2-Mutant (16 kDa). Recombinants PTD-Grb2-SH2 and PTD-Grb2-SH2-Mutant were expressed in $E$. coli after induction of IPTG. In order to obtain maximum soluble protein expression, we adjusted conditions, including temperature and time. Finally, we determined that a temperature of $33^{\circ} \mathrm{C}$ and induction time of $4 \mathrm{~h}$ was optimal for obtaining maximum amount of soluble protein (Fig. 1B). HisTrap ${ }^{\mathrm{TM}} \mathrm{Ni}^{++}$charged columns (Amersham Pharmacia, Piscataway, NJ, USA) were used to purify the His-tagged proteins. Purification products were analyzed by $15 \%$ SDS-PAGE and identified by western blot analysis (Fig. 1C).

Transduction of the recombinants into breast cancer cell line $M D A-M B-231$. An immunofluorescence assay with an anti-His-tag antibody was used to investigate whether the PTD-Grb2-SH2 and PTD-Grb2-SH2-Mutant could be transduced into living cells as designed. Purified PTD-Grb2-SH2 and PTD-Grb2-SH2-Mutant were added to cultured MDA-MB-231 cells for $2 \mathrm{~h}$ of incubation. Then, both recombinants were observed in the cells under a fluorescent microscope (Fig. 2). As shown in Fig. 2B and D, the recombinants including PTD-Grb2-SH2 and PTD-Grb2$\mathrm{SH} 2-$ Mutant were dispersed throughout the cytoplasm and 

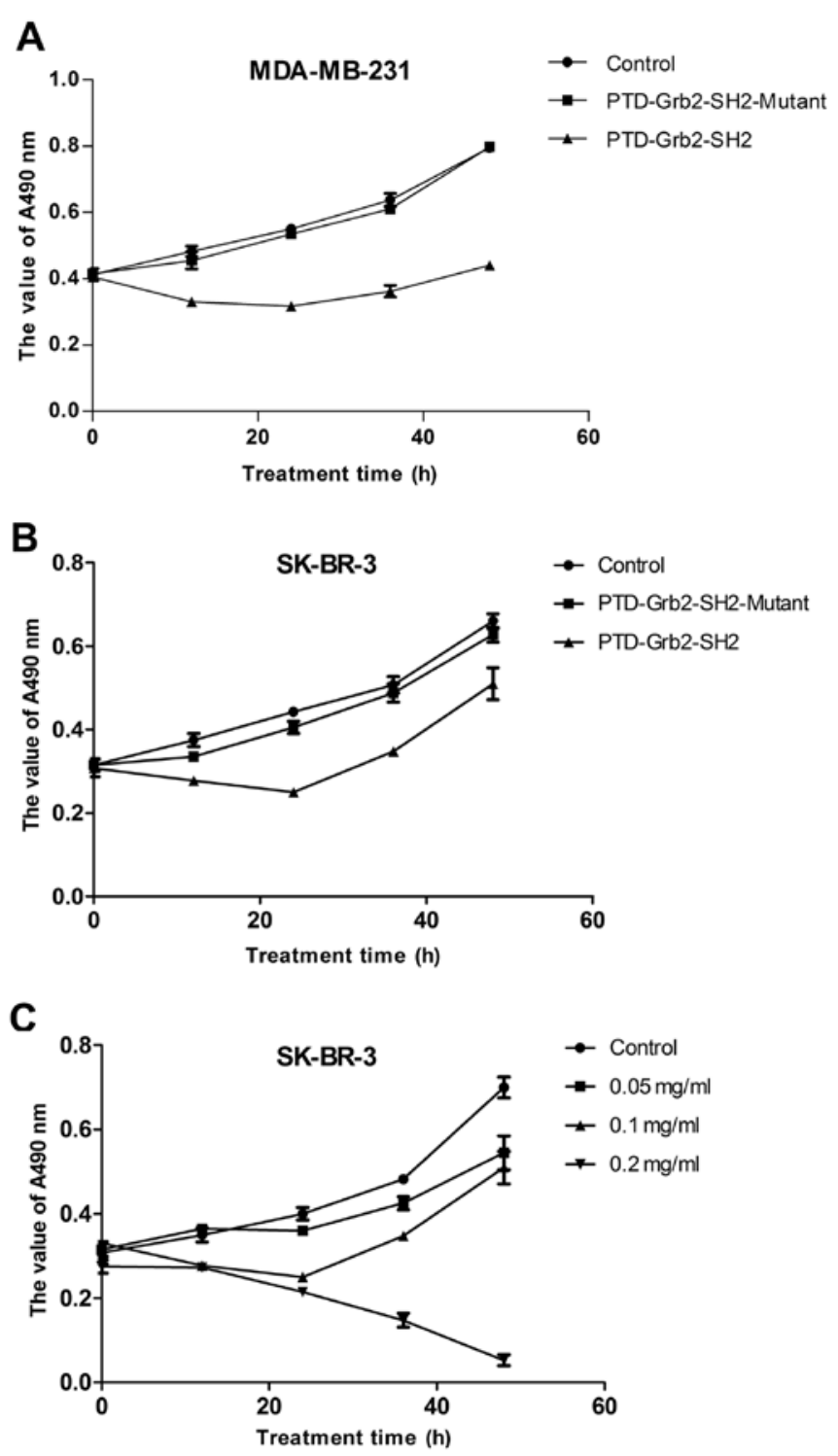

Figure 3. The growth curves of the breast cancer cell lines MDA-MB-231 and SK-BR-3. (A and B) The proliferation of the breast cancer cell lines MDA-MB-231 and SK-BR-3 were inhibited by PTD-Grb2-SH2 $(0.1 \mathrm{mg} / \mathrm{ml})$, but not PTD-Grb2-SH2-Mutant $(0.1 \mathrm{mg} / \mathrm{ml})$. (C) The inhibition effect of PTD-Grb2-SH2 is dose- and time-dependent.

mainly located in the nucleus, indicating HIV-1 TAT48-60 (GRKKRRQRRRPPQ) helped the target peptides to pass through both the cellular and nuclear membranes in living cells as reported (22).

Growth inhibition of PTD-Grb2-SH2 in the breast cancer cell lines $M D A-M B-231$ and $S K-B R-3$. The fresh recombinants of PTD-Grb2-SH2 and PTD-Grb2-SH2-Mutant were incubated with the breast cancer cell lines MDA-MB-231 and SK-BR-3 to investigate whether the new proteins could affect the proliferation of breast cancer cells in vitro. Fig. 3A and B show that the proliferation of MDA-MB-231 was inhibited by PTD-Grb2-SH2 in a time-dependent manner, but not by the mutant protein. Due to the similar inhibition effects of PTD-Grb2-SH2 in two different breast cancer cell lines, we chose the SK-BR-3 cells to look for the optimal concentration. Fig. $3 \mathrm{C}$ reveals that the recombinant PTD-Grb2-SH2 exhibited significant toxicity to breast cancer cells in a dose- and time-dependent manner in vitro. EdU identified the proliferation rates of MDA-MB-231 and SK-BR-3 cells. After incubation with PTD-Grb2-SH2 $(0.1 \mathrm{mg} / \mathrm{ml})$ for $12 \mathrm{~h}$, a decreased rate of cell proliferation was detected in MDA-MB-231 cells, compared to the untreated group $(24.6 \pm 1.4$ vs. $39.6 \pm 1.3 \%, \mathrm{P}<0.01)$. The proliferation rate of SK-BR-3 cells was also found to be decreased in the treated group, compared to the control group $(13.4 \pm 1.1$ vs. $37.6 \pm 2.2 \%$, $\mathrm{P}<0.01)$. However, there is no difference between the cells treated with PTD-Grb2-SH2-Mutant $(0.1 \mathrm{mg} / \mathrm{ml})$ and control in either breast cancer cell line (Fig. 4).

PTD-Grb2-SH2 induces apoptosis in MDA-MB-231 and $S K-B R-3$ cells. By light microscopy, after $12 \mathrm{~h}$ incubation with the fusion protein $(0.1 \mathrm{mg} / \mathrm{ml})$, many breast cancer cells began to shrink and lose their normal fibroblast-like shape compared with the untreated cells. We gathered the treated cells to be observed under an electron microscope. Characteristic forms associated with cellular apoptosis could be observed, including the shrinkage of cellular and nuclear membranes and the appearance of many high-density structures and vesicles in MDA-MB-231 and SK-BR-3 cells were observed. Many vesicles appeared in the cytoplasm and the chromosome condensed into armillary shapes and concentrated beneath karyotheca, resulting in crescent or ring shapes (Fig. 5). We identified these changes as apoptotic phenomena, suggesting that PTD-Grb2-SH2 may induce apoptosis in breast cancer cells. To further determine the cytotoxicity of PTD-Grb2-SH2 on cell proliferation, MDA-MB-231 and SK-BR-3 cells were exposed to PTD-Grb2-SH2 $(0.1 \mathrm{mg} / \mathrm{ml})$ for $12 \mathrm{~h}$. In order to differentiate this from necrosis and to confirm it as apoptosis, we performed fluorescein-conjugated Annexin V (Annexin V-FITC) flow cytometry. We quantitated the number of cells undergoing apoptosis. Our results showed that PTD-Grb2-SH2 induced apoptosis in $14.2 \%$ of MDA-MB-231 cells and $19.5 \%$ of SK-BR-3 cells, compared to the controls $(6.3$ and $11.3 \%$, respectively) (Fig. 6).

\section{Discussion}

Although the discovery and characterization of HER2 and herceptin have resulted in great progress in breast cancer treatment, many patients still eventually relapse. Consequently, there is an urgent need for additional therapeutic strategy other than HER2 signaling pathway. Grb2 is an important adapter protein and the first trigger for many cellular signal pathways involved in the processes of cell proliferation and mitogenesis (23). Blocking the interaction between pTyr-containing activated receptors and the $\mathrm{SH} 2$ domain of Grb2 is considered to be an effective and non-cytotoxic strategy in the development of new anti-proliferate agents due to its potential to shut down the Ras activation pathway (24). This makes the Grb2-SH2 domain an ideal target for breast cancer treatment.

In this study, we designed, constructed, expressed and purified a fusion protein that contained a PTD domain and a Grb2-SH2 domain. The PTD domain is considered to deliver proteins of more than $120 \mathrm{kDa}$ into living cells and tissues $(25,26)$. Fusion proteins containing HIV-1 TAT have been reported to be successfully transduced into tumor cells and 
A
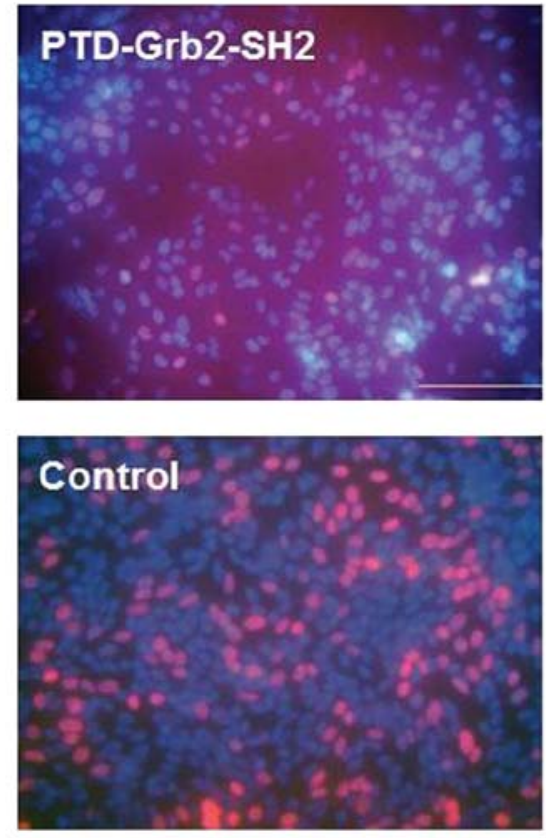

B
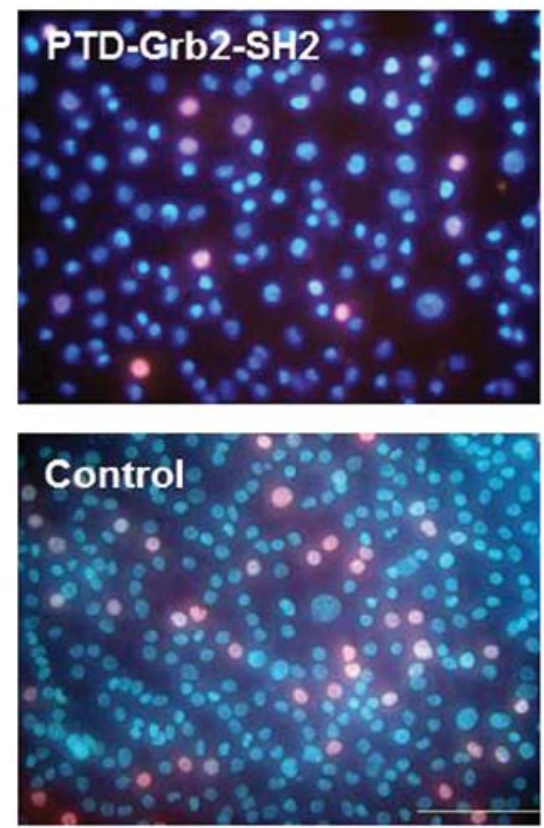
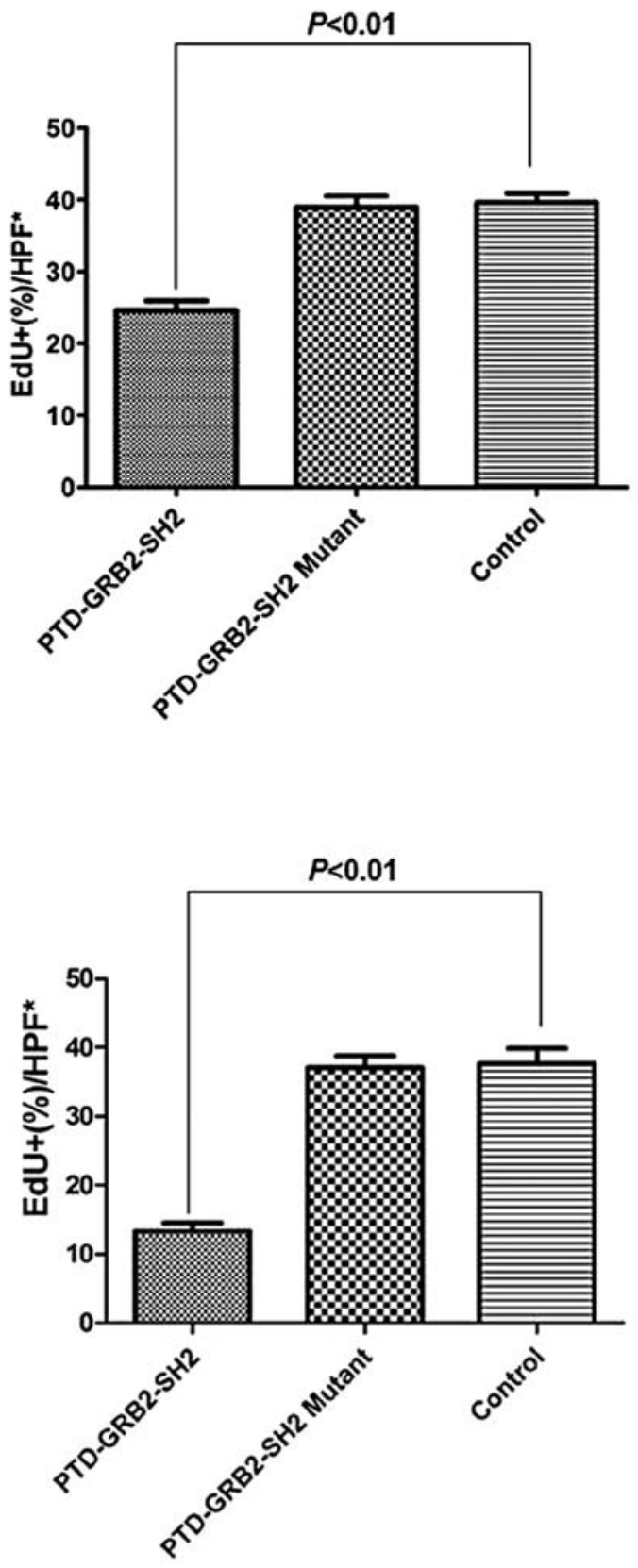

Figure 4. Inhibited cell proliferation of breast cancer cell lines MDA-MB-231 and SK-BR-3 after treated with PTD-Grb2-SH2 (0.1 mg/ml) for $12 \mathrm{~h}$. (A) After the treatment, a decreased rate of cell proliferation (24.6 $\pm 1.4 \%$ ) was detected in MDA-MB-231 cells, compared to the control cells $(39.6 \pm 1.3 \%)$. But there is no difference between the cells treated with PTD-Grb2-SH2-Mutant $(0.1 \mathrm{mg} / \mathrm{ml})$ and control. (B) A decreased rate of cell proliferation $(13.4 \pm 1.1 \%)$ was also detected in treated SK-BR-3 cells, compared to the control cells $(37.6 \pm 2.2 \%)$. No difference was found between the cells treated with PTD-Grb2-SH2-Mutant $(0.1 \mathrm{mg} / \mathrm{ml})$ and control. EdU labeling in cells is depicted as red fluorescence. Ratio of EdU positive cells to total number of cells (represented by blue nuclei stain) is a direct index of the number of proliferating cells. Significance of difference was calculated using Student's t-test. HPF*, high power field.

applied for anticancer therapy (27-29). We chose TAT ${ }^{48-60}$ (GRKKRRQRRRPPQ) as the PTD domain for transduction because this motif is the smallest one carrying PTD function without interfering with the target protein's function (22). Our data confirmed that both the fusion protein and mutant protein successfully passed through the cellular and nuclear membranes of living breast cancer cells with the help of PTD domain. TAT ${ }^{48-60}$ delivery is a convenient research tool to study the function of small target peptides or small proteins in living cells. In previous studies we used this PTD domain to successfully deliver SH3 domains into the leukemia cell line K562 and the hepatocarcinoma cell line HepG-2 (30,31). The Grb2$\mathrm{SH} 2$ domain is a highly preserved domain in both prokaryote and eukaryote, and the fusion proteins we designed are small proteins with molecular weight less than $20 \mathrm{kDa}$. Although the prokaryotic expression system we chose is not a theoretically proper expression system for a eukaryotic protein, we confirmed the right construction by automatic sequencing and 
A

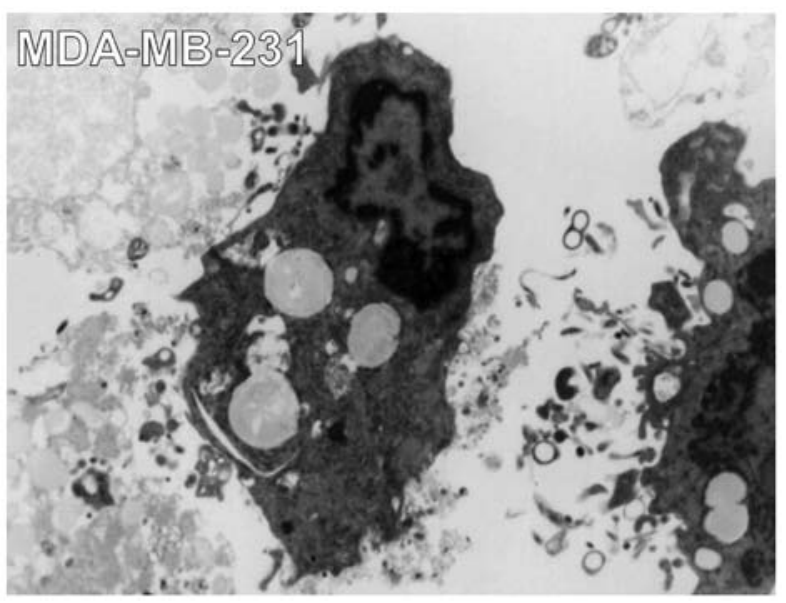

B

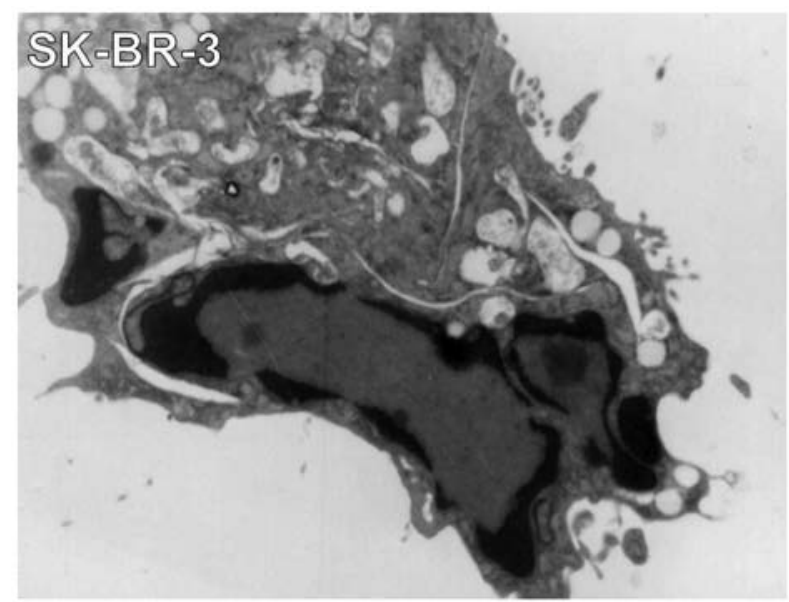

Figure 5. Ultra structure alterations of (A) MDA-MB-231 and (B) SK-BR-3 cells after $12 \mathrm{~h}$ of incubation with PTD-Grb2-SH2 (0.2 mg/ml) under an electron microscope. The apoptotic phenomena of MDA-MB-231 and SK-BR-3 cells were observed showing that chromosome condensed into armillary shapes and clung to the karyotheca.

A

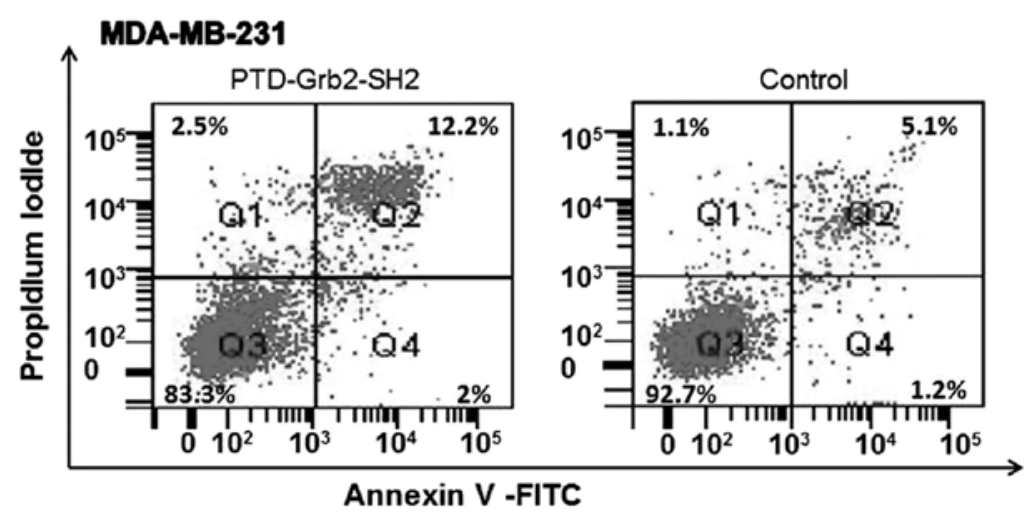

B

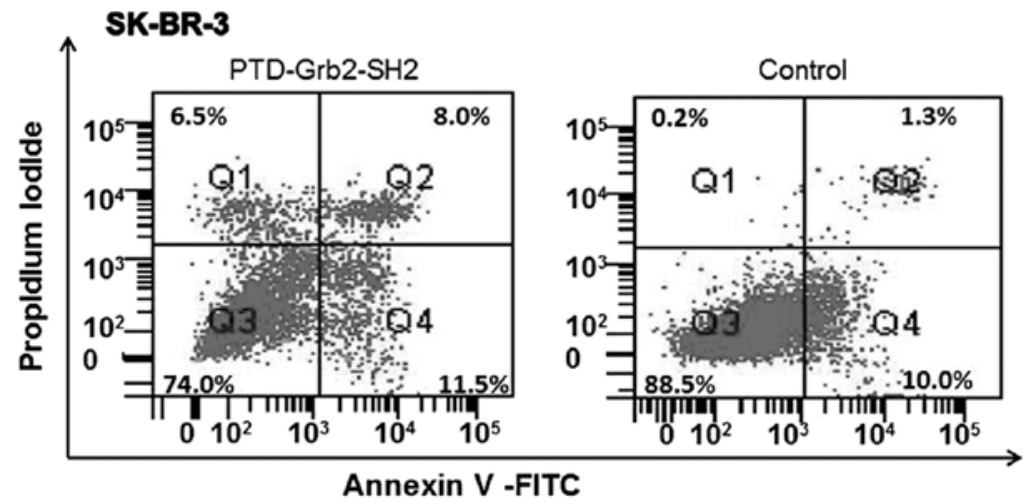

Figure 6. Using Annexin V-FITC, propidium iodide flow cytometry, PTD-Grb2-SH2 (0.1 mg/ml) induced cytotoxicity in human breast cancer cell lines and was confirmed as apoptosis. (A) Effect of PTD-Grb2-SH2 on MDA-MB 231 cells. (B) Effect of PTD-Grb2-SH2 on SK-BR3 cells. Q1, necrotic cells; Q2, cells at late stage of apoptosis; Q3, living cells; Q4, cells at early stage of apoptosis.

identified the target proteins by western blot analysis to prevent miss-construction and expression.

In function assay, we directly incubated the recombinants with HER2-negative MDA-MB-231 and HER2-positive SK-BR-3 to test the effects on the proliferation of breast cancer cells. Our data exhibited that PTD-Grb2-SH2 inhibited the proliferation of both breast cancer cell lines, but the PTD-Grb2-SH2-Mutant did not exert any inhibition to these cell lines, indicating the loss in basic sequence of Grb2-SH2 will result in dysfunction of the target protein. Our results also revealed that PTD-Grb2-SH2 exhibited significant toxicity to breast cancer cells in a dose- and time-dependent manner in vitro, which is an appropriate characteristic for anticancer drug developing. To date, many strategies have been used to inhibit the function of Grb2 in order to block crucial intracellular signals. Some studies have focused on designing and 
synthesizing phosphotyrosines or their mimics based upon space structures of Grb2 (32). Grb2 is made up of one SH2 domain surrounded by two $\mathrm{SH} 3$ domains. While both $\mathrm{SH} 2$ and SH3 domains possess a strong ability to recognize and specifically bind to their ligands, $\mathrm{SH} 2$ domains are the major known binding modules for tyrosine-phosphorylated proteins and are the prototype for protein-protein interactions that mediate the formation of multi-proteins complexes during signaling (33). Integrating these basic results with our data, we think that the strength and specificity of Grb2-SH2 for its ligands give this domain the ability of inhibiting Grb2 related signaling in living breast cancer cells.

We chose HER2-negative MDA-MB-231 and HER2positive SK-BR-3 in the function assay as Grb2 intermediates the mitogen-activated protein kinase (MAPK) pathway and also regulates receptor trafficking (34-36). It works with various RTKs, with EGFR being its major binding partner (37,38), which regulates many cell functions. Our data showed PTD-Grb2-SH2 inhibited HER2-negative and -positive breast cancer cells, indicating this fusion protein possesses non-specificity for single pathway. This is also the reason why we did not test the downstream molecules or pathways for this protein. There are too many molecules and pathways associated with Grb2 that remain to be elucidated in further experiments. Nevertheless, we still used electron microscopy and flow cytometry assay to identify the apoptotic phenomena induced by PTD-Grb2-SH2.

In conclusion, our study successfully constructed a target fusion protein expressing both PTD and Grb2-SH2 domains and showed that expression of the fusion protein resulted in growth inhibition and cell death in breast cancer cells regardless of HER2-phenotype. We proved that the TAT ${ }^{48-60}$ is a useful tool and could be capable of delivering outside proteins through the plasma membrane of living cells, and even delivering a protein directly to the cell nucleus. This technique will help us to demonstrate protein function in living cells. We also demonstrated that the $\mathrm{SH} 2$ domain is a highly conserved protein functional domain and can maintain its biological activity even when expressed in bacteria. The extent of inhibition depend on the concentration of the protein and the length of the time, moreover, it suggested that the protein might induce apoptosis in breast cancer cells. These results indicate that the PTD-Grb2-SH2 protein has the potential to be developed for treatment of breast cancer.

\section{Acknowledgements}

This study was supported by grant no. 30901457 from National Natural Science Foundation of China.

\section{References}

1. DeSantis C, Siegel R, Bandi P and Jemal A: Breast cancer statistics, 2011. CA Cancer J Clin 61: 409-418, 2011.

2. Harris AL, Nicholson S, Sainsbury JR, Farndon J and Wright C: Epidermal growth factor receptors in breast cancer: association with early relapse and death, poor response to hormones and interactions with neu. J Steroid Biochem 34: 123-131, 1989.

3. Sainsbury JR, Malcolm AJ, Appleton DR, Farndon JR and Harris AL: Presence of epidermal growth factor receptor as an indicator of poor prognosis in patients with breast cancer. J Clin Pathol 38: 1225-1228, 1985.
4. Nielsen TO, Hsu FD, Jensen K, et al: Immunohistochemical and clinical characterization of the basal-like subtype of invasive breast carcinoma. Clin Cancer Res 10: 5367-5374, 2004.

5. Slamon DJ, Godolphin W, Jones LA, et al: Studies of the HER-2/neu proto-oncogene in human breast and ovarian cancer. Science 244: 707-712, 1989.

6. Foster CS, Gosden CM and Ke Y: HER2/neu expression in cancer: the pathologist as diagnostician or prophet? Hum Pathol 34: 635-638, 2003

7. Rosen PP, Lesser ML, Arroyo CD, Cranor M, Borgen P and Norton L: Immunohistochemical detection of HER2/neu in patients with axillary lymph node negative breast carcinoma. A study of epidemiologic risk factors, histologic features, and prognosis. Cancer 75: 1320-1326, 1995.

8. Carlomagno C, Perrone F, Gallo C, et al: c-erb B2 overexpression decreases the benefit of adjuvant tamoxifen in early-stage breast cancer without axillary lymph node metastases. J Clin Oncol 14: 2702-2708, 1996.

9. Gusterson BA, Gelber RD, Goldhirsch A, et al: Prognostic importance of c-erbB-2 expression in breast cancer. International (Ludwig) Breast Cancer Study Group. J Clin Oncol 10: 1049-1056, 1992.

10. Bianco R, Melisi D, Ciardiello F and Tortora G: Key cancer cell signal transduction pathways as therapeutic targets. Eur J Cancer 42: 290-294, 2006.

11. Chardin P, Cussac D, Maignan S and Ducruix A: The Grb2 adaptor. FEBS Lett 369: 47-51, 1995.

12. Seger R and Krebs EG: The MAPK signaling cascade. FASEB J 9: 726-735, 1995.

13. Di Fulvio M, Henkels KM and Gomez-Cambronero J: Short-hairpin RNA-mediated stable silencing of Grb2 impairs cell growth and DNA synthesis. Biochem Biophys Res Commun 357: 737-742, 2007.

14. Machida $\mathrm{K}$ and Mayer BJ: The SH2 domain: versatile signaling module and pharmaceutical target. Biochim Biophys Acta 1747: $1-25,2005$.

15. Pawson T: Protein modules and signalling networks. Nature 373: 573-580, 1995 .

16. Mayer BJ, Jackson PK and Baltimore D: The noncatalytic src homology region 2 segment of abl tyrosine kinase binds to tyrosine-phosphorylated cellular proteins with high affinity. Proc Natl Acad Sci USA 88: 627-631, 1991.

17. Mayer BJ, Jackson PK, Van Etten RA and Baltimore D: Point mutations in the abl SH2 domain coordinately impair phosphotyrosine binding in vitro and transforming activity in vivo. Mol Cell Biol 12: 609-618, 1992.

18. Schwarze SR, Hruska KA and Dowdy SF: Protein transduction: unrestricted delivery into all cells? Trends Cell Biol 10: 290-295, 2000

19. Lindgren M, Hallbrink M, Prochiantz A and Langel U: Cell-penetrating peptides. Trends Pharmacol Sci 21: 99-103, 2000.

20. Liang Y, Sun Q, Jiang S, et al: Construction and expression of a vector containing protein transduction domain and bcr/abl fusion gene. Zhonghua Xue Ye Xue Za Zhi 23: 5-8, 2002 (In Chinese).

21. Laemmli UK: Cleavage of structural proteins during the assembly of the head of bacteriophage T4. Nature 227: 680-685, 1970.

22. Sangadala S, Okada M, Liu Y, Viggeswarapu M, Titus L and Boden SD: Engineering, cloning, and functional characterization of recombinant LIM mineralization protein-1 containing an N-terminal HIV-derived membrane transduction domain. Protein Expr Purif 65: 165-173, 2009.

23. Yarden Y and Sliwkowski MX: Untangling the ErbB signalling network. Nat Rev Mol Cell Biol 2: 127-137, 2001.

24. Lung FD and Tsai JY: Grb2 SH2 domain-binding peptide analogs as potential anticancer agents. Biopolymers 71 : $132-140,2003$.

25. Kim D, Jeon C, Kim JH, et al: Cytoplasmic transduction peptide (CTP): new approach for the delivery of biomolecules into cytoplasm in vitro and in vivo. Exp Cell Res 312: 1277-1288, 2006.

26. Schwarze SR, Ho A, Vocero-Akbani A and Dowdy SF: In vivo protein transduction: delivery of a biologically active protein into the mouse. Science 285: 1569-1572, 1999.

27. Tan M, Lan KH, Yao J, et al: Selective inhibition of ErbB2-overexpressing breast cancer in vivo by a novel TAT-based ErbB2-targeting signal transducers and activators of transcription 3-blocking peptide. Cancer Res 66: 3764-3772, 2006. 
28. Harada H, Kizaka-Kondoh S and Hiraoka M: Antitumor protein therapy; application of the protein transduction domain to the development of a protein drug for cancer treatment. Breast Cancer 13: 16-26, 2006.

29. Sorriento D, Campanile A, Santulli G, et al: A new synthetic protein, TAT-RH, inhibits tumor growth through the regulation of NFkappaB activity. Mol Cancer 8: 97, 2009.

30. Liang YJS, Han H, Liu L, Sun Q, Chen R, Wu R, Du J and Li Q: TAT PTD-BCR/ABL SH3 fusion protein induces the apoptosis of K562 leukemic cell line. J Fourth Mil Med Univ 23: 4, 2002.

31. Yin JK, Liang YM, He XL, et al: Fusion protein containing SH3 domain of c-Abl induces hepatocarcinoma cells to apoptosis. Hepatol Res 37: 454-463, 2007.

32. Ogura K, Shiga T, Yokochi M, Yuzawa S, Burke TR Jr and Inagaki F: Solution structure of the Grb2 SH2 domain complexed with a high-affinity inhibitor. J Biomol NMR 42: 197-207, 2008.
33. Dierck K, Machida K, Mayer BJ and Nollau P: Profiling the tyrosine phosphorylation state using $\mathrm{SH} 2$ domains. Methods Mol Biol 527: 131-155, 2009.

34. Jiang X, Huang F, Marusyk A and Sorkin A: Grb2 regulates internalization of EGF receptors through clathrin-coated pits. Mol Biol Cell 14: 858-870, 2003.

35. Yamazaki T, Zaal K, Hailey D, Presley J, Lippincott-Schwartz J and Samelson LE: Role of Grb2 in EGF-stimulated EGFR internalization. J Cell Sci 115: 1791-1802, 2002.

36. Burke P, Schooler K and Wiley HS: Regulation of epidermal growth factor receptor signaling by endocytosis and intracellular trafficking. Mol Biol Cell 12: 1897-1910, 2001.

37. Schulze WX, Deng L and Mann M: Phosphotyrosine interactome of the ErbB-receptor kinase family. Mol Syst Biol 1: 2005.0008, 2005.

38. Seiden-Long I, Navab R, Shih W, et al: Gab1 but not Grb2 mediates tumor progression in Met overexpressing colorectal cancer cells. Carcinogenesis 29: 647-655, 2008. 